\title{
La promoción del deporte a través de la felicidad del deportista federado en Kárate
}

The Promotion of Sports Through the Happiness of Affiliated Sportspeople in Kárate

Pedro Cuesta-Valiño

Doctor en Ciencias Económicas y Empresariales Universidad de Alcalá, (Madrid, España) pedro.cuesta@uah.es

Cristina Loranca-Valle

Doctoranda en Economía y Administración de Empresas

Universidad de Alcalá (Madrid, España)

cristina.loranca@uah.es

Estela Núñez-Barriopedro

Doctora en Economía y Administración de Empresas Universidad de Alcalá (Madrid, España)

estela.nunezb@uah.es
Cuesta Valiño, P., Loranca-Valle, C. y Nuñez-Barriopedro, E. (2020)

La promoción del deporte a través de la felicidad del deportista federado en Kárate

Revista Internacional de Investigación en Comunicación aDResearch ESIC. № 21 Vol 21

Primer semestre, enero-junio 2020 · Págs. 48 a 69

https://doi.org/10.7263/adresic-021-03 
RESUMEN

\section{Clasificación JEL: M310}

\section{Palabras clave:}

Felicidad, satisfacción, calidad percibida, deporte, kárate
Objetivo: El principal objetivo del presente trabajo es primeramente analizar las causas de la escasa variación en el número de licencias deportivas en de los 10 últimos años y en consecuencia aportar estrategias de captación y fidelización del federado. Una de las novedades de este trabajo es que se analizan las principales variables que afectan a la felicidad del consumidor. Así, las organizaciones pueden gestionar estas variables con el objeto de hacer más felices a sus federados, lo que aumentaría su fidelidad y atraería a nuevos miembros.

Diseño/Metodología: Primeramente, se ha realizado un estudio exploratorio con revisión de la literatura, seguida de un estudio correlacional con información primaria mediante encuesta. Para ello, se analizan las variables que influyen en la felicidad del deportista obteniendo una muestra de 601 deportistas federados. El trabajo de campo ha sido llevado a cabo en las distintas federaciones de kárate autonómicas de España.

Resultados: La revisión de la literatura indica que las principales variables explicativas de la felicidad son la calidad percibida y la satisfacción. Asimismo, los resultados del estudio empírico confirman que las cuatro variables calidad del servicio, la satisfacción y la confianza analizadas influyen positivamente en la felicidad. Así, las federaciones pueden gestionar enfocadas en maximizar la felicidad de sus miembros y relacionar la felicidad con los servicios de la federación.

Limitaciones/Implicaciones: Una de las principales limitaciones es que esta investigación se ha llevado sobre una disciplina deportiva específica, el kárate. Por lo que en futuras líneas de investigación se puede extrapolar a otras federaciones que generen nuevas perspectivas.

Original/Contribución: Las federaciones deportivas difunden el deporte en la sociedad. Sin embargo, el número de deportistas federados ha aumentado escasamente en los últimos años. Como consecuencia, el presente trabajo contribuye a explicar cómo se pueden gestionar las federaciones para conseguir consumidores fieles junto con un aumento más considerable de federados.

\section{ABSTRACT}

\author{
JEL Classification: \\ M310 \\ Key words: \\ Happiness, \\ satisfaction, \\ perceived quality, \\ sport, \\ karate
}

Objective: The main objective of the present work is first to analyze the causes of the scarce variation in the number of sports licenses in the last 10 years and consequently contribute strategies to attract and retain the affiliated member. One of the novelties of this work is that the main variables that affect consumer happiness are analyzed. Thus, organizations can correctly manage these variables in order to make their members happier. Happier people are more likely to continue in the federation and attract new members.

Design/Methodology: Firstly, an exploratory study was carried out with a review of the information, followed by a correlational study with primary information through a survey. For this, the variables that influence athletes' happiness are analyzed, obtaining a sample of 601 affiliated athletes. The fieldwork was carried out in the various autonomous karate federations in Spain.

Results: The data reveals that the main explanatory variables of happiness are perceived quality and satisfaction. Likewise, the results of the empirical study confirm that the variables analyzed for service quality, satisfaction and trust positively influence happiness. In this way, managers of sports federations can make decisions focused on maximizing the happiness of their members and creating communication strategies that connect the happiness and services of the federation more effectively.

Limitations/Implications: One of the main limitations is that this research has been carried out on a specific sport discipline, karate. So in future lines of research you can extrapolate to other federations that generate new perspectives.

Originality / Contribution: One of the main objectives sports federations have is the promotion and dissemination of sport in society. However, the real situation is that the number of federated athletes has increased scarcely in recent years. As a result of this situation, this study aims to contribute to explaining to the managers of sports federations how they can develop more creative and effective communication campaigns in hope of obtaining loyal consumers along with a more considerable increase in members. 


\section{Introducción}

Las federaciones deportivas tienen como fin principal la promoción y el impulso del deporte («Supreme Council for Sports in Spain. Ministry of Education, Culture and Sports,» 2007). Aunque son organismos privados, es la Administración Pública quien las concede el poder de actuación como órgano para el impulso del deporte de alto nivel y alto rendimiento. De esta manera, las federaciones están subvencionadas por la Administración, y es esta la que les otorga las principales dotaciones económicas con el propósito de maximizar el nivel deportivo de España internacionalmente («Supreme Council for Sports in Spain. Ministry of Education, Culture and Sports,» 2007). Por tanto, una de las finalidades de las federaciones deportivas es conseguir el mayor número de federados para conseguir entre estos deportistas éxitos para España a nivel internacional.

Por lo que respecta al número de federados, se ha visto que durante los últimos 10 años el número no ha aumentado, aunque se puede percibir cierto crecimiento en los últimos dos años. Pero este incremento resulta apenas perceptible si se amplía la línea temporal estudiada a 10 años.

Además, aunque es innegable que el deporte está produciendo un interés creciente en la población en los últimos años, sin embargo, a pesar de este interés en la actividad física y el bienestar físico, el número de miembros de organizaciones deportivas sin ánimo de lucro no han crecido de forma proporcional a este interés (Wemmer $\&$ Koenigstorfer, 2016). Las empresas deportivas con ánimo de lucro se han introducido en el mercado con mucha fuerza y cada vez atraen a más clientes, se están llevando la mayor parte de la cuota de mercado y las organizaciones no lucrativas como las federaciones no están sabiendo como plantarle cara a este incremento de la competencia (Smith \& Stewart, 2010).
Teniendo en cuenta este contexto, el objetivo del presente trabajo pretende analizar algunas de las causas por la cuales apenas se ha producido variación en el número de licencias deportivas de los 10 últimos años en los que se han recogido los datos. Las federaciones, al ser, organizaciones sin ánimo de lucro no buscan la obtención de beneficios, su principal finalidad es la promoción y divulgación del deporte, es decir, su finalidad es la de aumentar el número de federados lo máximo posible. Dado que el incremento no es lo que se podría esperar, entre otras causas, es posible que no estén sabiendo desarrollar campañas de comunicación efectivas. Para poder llevar a cabo el objetivo se analizarán las principales variables que afectan a la felicidad del consumidor, pues si las organizaciones gestionan de forma correcta estas variables podrían hacer más felices a sus federados. Los empleados felices tienen más probabilidades de continuar en la federación y de atraer a nuevos miembros. La felicidad de los federados, entre otras causas, contribuiría a que las federaciones alcanzasen su objetivo de promoción e impulso del deporte.

En este sentido, algunos autores han confirmado en sus investigaciones que el consumo como espectador deportivo (Hallmann, Breuer, $\& \mathrm{Ku}, 2013$; Jang, Ko, Wann, \& Kim, 2017), las vacaciones deportivas o la asistencia a eventos deportivos (Nicolao, Irwin, \& Goodman, 2009), o incluso la participación como atletas en eventos (Huang \& Humphreys, 2012; Theodorakis, Kaplanidou, \& Karabaxoglou, 2015) están muy relacionados con la felicidad e incrementan los niveles de esta.

El deporte y la felicidad están muy relacionados. Las personas han estado persiguiendo la felicidad desde el principio de los tiempos. El logro de los objetivos es una de las razones por las que los humanos experimentan la felicidad 
(Veenhoven, 2006). Enfocando esta afirmación en el contexto de esta investigación, alcanzar los objetivos deportivos puede conllevar a conseguir la felicidad. Además, el deporte está asociado a la vida saludable. De hecho, la falta de ejercicio físico y el sedentarismo está vinculada con algunos trastornos y enfermedades (Álvarez, 2007). Entre otras, esta es la razón por la que los gobiernos están interesados en el fomento y la promoción del deporte. Pero aparte de la salud existen otras razones: mejora de la forma física, frenar la obesidad, la lucha contra el crimen, y proveer valores a la juventud (Huang \& Humphreys, 2012; Hur, Ko, \& Valacich, 2011; Schöppe, Bauman, \& Bull, 2014). Y por esta razón el gobierno de España les otorga a las federaciones deportivas españolas el desarrollo y la organización del deporte de alto nivel y rendimiento. De esta manera, la administración pública financia a las federaciones para que estas se encarguen de elevar el nivel deportivo en España y en el extranjero (Consejo Superior de Deportes, 2019).

La felicidad que produce el deporte puede ser la fórmula adecuada que permita atraer a más federados y mantener a los que ya lo son, si se consiguen desarrollar planes de comunicación dirigidos al público objetivo con la conexión entre la felicidad y las ventajas de estar federado. Si esta relación es correcta, estos planes de comunicación producirán un incremento en el número de deportistas federados. Por ello, en esta investigación vamos a estudiar las variables que condicionan la felicidad del federado deportivo. El objetivo final es ayudar a que los directivos de las federaciones deportivas tengan más herramientas y conocimiento para mejorar la felicidad de los deportistas. Y así la felicidad ayudará a promocionar el deporte y a incrementar el número de miembros federados. Además, si los directivos tienen un mayor conocimiento de las razones que producen felicidad a sus deportistas podrán plantear estrategias de comunicación más eficientes y más acordes a las necesidades de todos sus miembros. En este estudio analizamos el caso concreto de las federaciones deportivas de kárate a nivel nacional.

\section{Revisión bibliográfica Felicidad}

La felicidad produce una serie de emociones y sensaciones, que cualquier persona sana mentalmente desearía mantener el mayor tiempo posible (Vasconcelos, 2008).

Algunos autores conceptualizan la felicidad como un estado psicológico positivo derivado de una experiencia buena, agradable, y/o satisfactoria (Ahuvia, 2008; Jang et al., 2017; Lyubomirsky, King, \& Diener, 2005). La felicidad se puede mirar a través de dos perspectivas, una de ellas se refiere a un momento dado en el tiempo y la otra se refiere a una duración continua; la primera es consecuencia de una situación o de una experiencia positiva particular mientras que la segunda se produce a raíz de un estado psicológico positivo general que es acumulativo en el tiempo (Lyubomirsky, Sheldon, \& Schkade, 2005).

La literatura estudiada confirma en numerosos estudios que la felicidad y la lealtad están positivamente relacionadas, Aksoy et al. (2015) confirman en su trabajo empírico que tanto la lealtad concreta como la abstracta influyen en la felicidad, de hecho, el balance óptimo entre ambas contribuirá a maximizar la felicidad general. La satisfacción del cliente no implica la felicidad del cliente, pero si se pretender conducir al cliente hacia la lealtad hay que buscar su felicidad (Khan $\&$ Hussain, 2013) antes que enfocarse en su satisfacción que es lo que se ha estado dando durante los últimos 50 años (Easterlin, 2001). 
La felicidad es considerada una variable multifacética ya que está compuesta el item de afecto positivo, de la ausencia de afecto negativo y de juicios sobre la satisfacción con la vida (Diener, Lucas, \& Oishi, 2002).

La calidad de vida y la felicidad se han relacionado en muchas ocasiones, y no solamente eso, sino que cuando una buena parte de la población se siente feliz, la calidad de vida de esa población será buena (Veenhoven, 1997). Las personas felices suelen tender a tener vidas prósperas, de manera externa e interna (Sheldon, Lyubomirsky, \& D., 2002). En la teoría sobre felicidad, algunos autores defienden que la felicidad de una persona concreta depende de una serie de elementos relacionados con sentimientos positivos como son las relaciones sociales, el trabajo y el desempleo, el ocio, el dinero, la clase, la cultura, la personalidad, la alegría, la satisfacción con la vida, la edad, el sexo, la mejora de la salud, etc. Esto viene a resumirse en las circunstancias generales de la vida las cuales repercuten en la felicidad de las personas (Argyle, 1994; Vasconcelos, 2008).

\section{Calidad Percibida}

La definición de calidad percibida que más autores han defendido se basa en las discrepancias entre las percepciones del consumidor sobre un determinado bien o servicio ofrecido y las expectativas sobre dicho producto (Boulding, Kalra, Staelin, \& Zeithaml, 1993; Hennig-Thurau, Gwinner, \& Gremler, 2002; Parasuraman, Zeitha$\mathrm{ml}, \&$ Berry, 1988). Si suponemos igualdad de percepciones, cuantas más altas sean las expectativas del consumidor menor va a ser la calidad percibida (Parasuraman et al., 1988).

La calidad percibida es el juicio que hace el consumidor sobre la excelencia general de una entidad (Zeithaml, 1987) y es diferente de la ca- lidad objetiva (Parasuraman et al., 1988). El concepto de calidad tiene dos dimensiones: una mecánica y otra humanística. La dimensión mecánica es relativa a las características objetivas del intercambio, por otro lado, la dimensión humanística de la calidad se refiere a la respuesta subjetiva de las personas frente a un caso específico y dependerá del juicio de cada individuo (Holbrook \& Corfman, 1985).

En el caso de las federaciones deportivas, como se trata de servicios, los clientes tienen un mayor riesgo percibido en la relación de intercambio. Esto se debe a las principales características de los servicios: la intangibilidad y la variabilidad. Esta es la razón por la que la credibilidad es uno de los aspectos que más relevancia tiene para el consumidor en el momento de evaluar la calidad percibida (Javalgi \& Moberg, 1997). Parasuraman et al. (1988) defienden que existen 10 potenciales atributos de la calidad de los servicios que se superponen unos a otros. Estos atributos son: la tangibilidad; la fiabilidad; la capacidad de respuesta, la comunicación; la credibilidad; la seguridad; la competencia; la cortesía; el conocimiento del consumidor; y la accesibilidad.

\section{Satisfacción}

La satisfacción se puede definir de diferentes formas, la satisfacción de una acción concreta y la satisfacción acumulada (Boulding et al., 1993). La satisfacción acumulada se define como la evaluación global basada en la suma de las transacciones de un determinado producto (Eugene Anderson, Fornell, \& Lehmann, 1994; Fornell, 1992).

Otros autores definen la satisfacción como la reacción sentimental del consumidor ante las diferencias percibidas entre la prestación del servicio y las expectativas (Andreassen, 2000; Oliver, 1980). Sin embargo, esta definición po- 
dría suponer una confusión por sus semejanzas con la definición de Calidad Percibida. La realidad es que ambas variables son distintas, encontrándose la mayor diferencia en que la satisfacción es una especie de actitud, una evaluación en el largo plazo, y la calidad percibida es la medida de una transacción específica (Parasuraman et al., 1988). Andreassen (2000) vincula la satisfacción con el hecho de suministrar lo que se está buscando hasta el punto de que se pueda alcanzar esta.

\section{Confianza}

En la literatura podemos encontrar diversos autores que coinciden en que para tener éxito en las relaciones la confianza es una pieza clave (Berry, 1995; Dwyer, Schurr, \& Oh, 1987; Moorman, Deshpandé, \& Zaltman, 1993). La confianza en una empresa u organización deriva de la seguridad que tiene el consumidor en la integridad y la calidad que le ofrece el proveedor (Garbarino \& Johnson, 1999; Hennig-Thurau, Langer, \& Hansen, 2001; Moorman et al., 1993; Morgan \& Hunt, 1994). La confianza implica la creencia por parte de consumidor de que el proveedor ejecutará acciones satisfactorias (Erin Anderson \& Weitz, 1989). En las relaciones entre las compañías y el consumidor es más importante la ganancia psicológica que proveen la confianza y la seguridad que un trato especial o beneficios sociales originados de la relación (Gwinner, Gremler, \& Bitner, 1998). La confianza se refiere a la implicación en un proceso que ha sido muy bien pensado y cuidadosamente repasado, mientras que, cuando hablamos del afecto a una marca, estamos hablando más bien de algo espontaneo, más impulsivo y menos razonado (Chaudhuri \& Holbrook, 2001). La confianza tiene dos dimensiones: una afectiva y otra cognitiva (Johnson \& Grayson, 2005).

\section{Relación entre calidad percibida y felicidad}

Una clara forma de enriquecer de forma positiva la psicología del consumidor es a través de la mejora de la calidad del servicio (Andreasen, 1994).

En la literatura encontramos varios autores que confirman la relación existente entre calidad percibida y felicidad (Gong \& Yi, 2018; Sato, Jordan, \& Funk, 2014; Theodorakis et al., 2015; Loranca Valle, Cuesta-Valiñoy Núñez-Barriopedro, 2019).

Theodorakis et al. (2015) analiza la relación que existe entre la calidad percibida y la satisfacción sobre la felicidad que puede proporcionar el consumo de eventos deportivos, su estudio se basa en las afirmaciones de Sato, Jordan, \& Funk (2014), que afirmaron que «el ocio físicamente activo puede mejorar la calidad de vida de los participantes al proporcionar experiencias positivas a través de la participación en eventos». En el contexto de los eventos deportivos, Theodorakis et al. (2015) señalan 3 tipos de calidad del servicio: la calidad enfocada en los resultados, la calidad en la interacción y la calidad del entorno físico. Solamente la calidad de los resultados tiene influencia sobre la felicidad.

La satisfacción también está muy relacionada con la calidad percibida, algunas veces, esta actúa como una variable mediadora entre esta y la felicidad, como se produce en el estudio de Gong \& Yi (2018), en el que se miden varios factores de las percepciones de la calidad general del servicio como podría ser el entorno, el desarrollo o la entrega de servicio en cuestión. Estas tres variables explican la satisfacción que a su vez explica la lealtad y la felicidad.

Considerando las aportaciones de la teoría sobre la relación existente entre calidad percibida y felicidad planteamos las siguientes hipótesis: 
H1: La calidad percibida tiene una influencia positiva sobre la satisfacción.

H2: La calidad percibida tiene una influencia positiva sobre la felicidad.

\section{Relación entre satisfacción y felicidad}

La satisfacción con la vida y la felicidad son dos conceptos distintos, pero Haller \& Hadler, (2006) proponen en sus investigaciones que los elementos macrosociales, como la riqueza económica de un país, la distribución de la renta, la amplitud del estado del bienestar, y el liberalismo político influyen tanto en ambas.

Cuando un servicio se lleva a cabo varias veces en el tiempo, las experiencias procedentes de estos intercambios forman el núcleo de la satisfacción del consumidor y al mismo tiempo esto conlleva a la felicidad del consumidor (Dagger $\&$ Sweeney, 2006).

Además, la satisfacción del consumidor se puede producir en cualquier momento en la vida, lo que también conduce a la felicidad (Sweeney, Danaher, \& Mccoll-kennedy, 2015). Esto podría estar relacionado con la teoría de abajo a arriba sobre la felicidad del consumidor, la cual determina que la satisfacción del consumidor, producida a partir de un servicio concreto, se expande hacia arriba a la satisfacción global con el servicio, y esta, a su vez se expande hacia la felicidad (Gong \& Yi, 2018).

Los resultados obtenidos de la literatura revisada sobre felicidad y satisfacción nos conducen a plantearnos la siguiente hipótesis:

H3: La satisfacción afecta positivamente a la felicidad.

\section{Relación entre confianza y felicidad}

Los trabajos que estudian la relación existente entre la confianza y la felicidad se han tratado principalmente a partir de datos agregados, tanto a nivel regional como nacional (Barra, Pressgrove,
\& Torres, 2018; Bartolini \& Mikucka, 2017; Tokuda, Fujii, \& Inoguchi, 2010), atendiendo a la dimensión geográfica.

Por su parte, Bartolini \& Mikucka (2017) estudian la relación que existe entre el bienestar subjetivo o la felicidad y la confianza social en el este de Europa, ellos concluyen que la confianza no influye sobre la felicidad a corto plazo, y por el contrario, en el medio o largo plazo, la confianza influye sobra la felicidad de igual forma que el crecimiento económico influye sobre esta. Otro estudio que analiza la relación entre la felicidad y la confianza de los japoneses, se defiende que la relación entre la confianza y la felicidad depende del contexto que se esté analizando (Barra et al., 2018).

La confianza puede ser una pieza clave en la disminución de potenciales influencias negativas en la felicidad las cuales pueden llegar a aparecer cuando el sistema legal no funciona bien (Barra et al., 2018). Las personas que se encuentran en países con mayores gados de confianza social agregada pueden tener más posibilidades de ser felices que las que se encuentran en países con un menor nivel de confianza (Tokuda et al., 2010).

A partir de las líneas de pensamiento de la literatura estudiada sobre felicidad y confianza, proponemos la siguiente hipótesis:

H4: La confianza afecta positivamente sobre la felicidad.

\section{Relación entre relaciones sociales y felicidad}

La satisfacción y la felicidad no se pueden alcanzar si se olvidan las relaciones sociales o si se deja de lado la sociedad (Haller \& Hadler, 2006). Las personas siempre han creído que la estructura social y las instituciones sociales les ofrecen la posibilidad de obtener una larga vida y felicidad (Boudon, 2002). Haller \& Hadler (2006) demuestran en su investigación su hipótesis sobre que existen ciertas relaciones sociales, las cuales 
en determinadas condiciones podrían producir felicidad o, por lo contrario, infelicidad.

Algunos estudios deportivos demuestran que las relaciones sociales afectan a la felicidad del seguidor deportivo utilizando la identificación con el equipo como variable mediadora: la relación con otros fans puede potenciar la identificación con el equipo y de igual forma influir sobre la felicidad (Jang et al., 2017). De hecho, los individuos sienten mayor felicidad al realizar actividades que proporcionan contactos sociales positivos y de alta calidad con otros (Haller \& Hadler, 2006; Jang et al., 2017)

En la literatura encontramos varios autores que están de acuerdo en que la amistad y la felicidad están positivamente relacionadas (Baldassare, Rosenfield, \& Rook, 1984; Diener \& Seligman, 2002; Gladow \& Ray, 1986).

Hay algunos autores que han investigado como la amistad influye en la felicidad, para ello analizan varios aspectos de esta como podrían ser los conflictos y la calidad (Demir \& Urberg, 2004; Demir \& Weitekamp, 2007; Mathur, 2004). Demir \& Weitekamp (2007) confirman empíricamente que ambos aspectos de la amistad afectan a la felicidad teniendo en cuenta estas otras dos variables: la personalidad y el género.

Considerando estas líneas de pensamiento, se plantea la siguiente hipótesis:

H5: Las relaciones sociales afectan positivamente sobre la felicidad que produce hacer deporte.

\section{Metodología de la investigación}

La población elegida para el desarrollo de este estudio son los federados en kárate españoles. Las federaciones deportivas se caracterizan por ser organizaciones muy especiales, su singularidad reside en que, a pesar de ser organizaciones privadas sin ánimo de lucro, actúan como agentes colaboradores de la Administración Pública y parte de sus actividades principales es realizar funciones propias de la Administración Pública. Las federaciones deportivas, como tienen tanto carácter privado como público a la vez, se las considera organizaciones mixtas. El objetivo principal de las federaciones es el de promover e impulsar el deporte a su máximo exponente («Supreme Council for Sports in Spain. Ministry of Education, Culture and Sports,» 2007). Para poder llevar a cabo ese objetivo, la Administración Pública las dota como órgano encargado de desarrollar el deporte de alto rendimiento y de alto nivel, y les aporta recursos económicos para su consecución(«Supreme Council for Sports in Spain. Ministry of Education, Culture and Sports,» 2007).

España cuenta con un total de 66 federaciones en todas las disciplinas deportivas, que en total suman 3.761.498 licencias deportivas en el año 2017. En el año 2008, se llegó a alcanzar el número de 3.394.384 individuos de licencias federadas. Por lo que se puede observar que se ha producido un aumento durante estos últimos diez años, si bien es cierto, el crecimiento lineal se ha mantenido constante pero es apenas perceptible, especialmente teniendo en cuenta la variación producida en la población española (Subdirección General de Estadística y Estudios Secretaría General Técnica y Deporte, 2015). Entre todas las federaciones deportivas españolas se ha elegido la de la disciplina de kárate por ser un caso destacado por el bajo crecimiento anual de las licencias deportivas.

Los datos se han obtenido a través de fuentes primarias mediante encuesta enviada a las federaciones autonómicas en la disciplina de kárate. Para una población de 72910 federados en karate (anuario de estadísticas deportivas, 2019) se ha obtenido una muestra de 601 individuos, con un error de muestreo de 4,06 al 95,5\% de confianza siendo $p=0,5 ; q=0,5$. El método de mues- 
treo utilizado ha sido por conveniencia de bola de nieve, ya que los propios federados respondieron voluntariamente y distribuyeron la encuesta a sus compañeros. Los datos obtenidos se han recogido de 11 federaciones autonómicas de 19 existentes. El trabajo se campo se ha implementado durante el primer trimestre de 2019. Entre las características de la muestra, el 71\% son hombres y el 29\% mujeres, todos ellos federados de la disciplina deportiva kárate. En la muestra se encuentran individuos de diversas edades, aunque el grupo con mayor participación es el que se encuentra entre los 45 y los 64 años, con una mayoría que lleva más de 20 años federados.

Las variables latentes tienen un Coeficiente alfa de Cronbach por encima del 0,8 lo que muestra consistencia interna. Concretamente, para la Calidad percibida es 0,915, satisfacción 0,8273 , confianza 0,9496 y felicidad 0,892. Asimismo, (ver tabla 1) se pueden ver los indicadores, usados en la encuesta, basados en la literatura. Cada uno de los indicadores ha seguido una escala de Likert de 5 puntos de total desacuerdo a total acuerdo.

\section{Tabla 1 - Variables e indicadores del estudio empírico}

\begin{tabular}{|c|c|c|c|}
\hline Variable & $\begin{array}{l}\text { Coeficiente } \\
\text { alfa de } \\
\text { Cronbach }\end{array}$ & Indicador & Literatura \\
\hline \multirow{5}{*}{$\begin{array}{l}\text { Calidad } \\
\text { percibida }\end{array}$} & \multirow{5}{*}{0,915} & $\begin{array}{l}\text { Mi federación proporciona un ambiente agradable para realizar } \\
\text { las actividades que organiza }\end{array}$ & \multirow{4}{*}{$\begin{array}{l}\text { (Parasuraman et al., 1988; } \\
\text { Su et al., 2016) }\end{array}$} \\
\hline & & $\begin{array}{l}\text { Las actividades de mi federación (campeonatos, cursos, } \\
\text { entrenamientos, etc.) se desarrollan de manera eficiente }\end{array}$ & \\
\hline & & $\begin{array}{l}\text { Los empleados de mi federación me atienden de forma eficaz } \\
\text { mis consultas }\end{array}$ & \\
\hline & & $\begin{array}{l}\text { Entiendo que mi federación cobra un precio justo por las } \\
\text { actividades en las que yo participo }\end{array}$ & \\
\hline & & $\begin{array}{l}\text { Los servicios y actividades que ofrece mi federación en global } \\
\text { los considero de calidad }\end{array}$ & $\begin{array}{l}\text { (Deng, Lu, Wei, \& Zhang, 2010; } \\
\text { Shin \& Kim, 2008) }\end{array}$ \\
\hline \multirow{3}{*}{ Satisfacción } & \multirow{3}{*}{0,8273} & $\begin{array}{l}\text { Estoy satisfecho con las actividades/servicios que ofrece mi } \\
\text { federación }\end{array}$ & $\begin{array}{l}\text { (Maxham \& Netemeyer, 2002; } \\
\text { Su, Swanson, \& Chen, 2016) }\end{array}$ \\
\hline & & Creo que es bueno para mi estar federado & \multirow{2}{*}{ (Deng, Lu, Kee, \& Zhang, 2010) } \\
\hline & & Estoy contento de estar federado en mi federación & \\
\hline \multirow{3}{*}{ Confianza } & \multirow{3}{*}{0,9496} & $\begin{array}{l}\text { Mi federación es honesta con todos federados } \\
\text { Mi federación se preocupa de los federados a los que prestan } \\
\text { sus actividades }\end{array}$ & $\begin{array}{l}\text { (Deng, Lu, Wei, \& Zhang, 2010; } \\
\text { Gefen, Karahanna, \& Straub, } \\
\text { 2003) }\end{array}$ \\
\hline & & $\begin{array}{l}\text { Mi federación tiene un comportamiento comprometido con } \\
\text { los federados a los que prestan sus actividades }\end{array}$ & $\begin{array}{l}\text { (Bonsón Ponte et al, 2015; } \\
\text { Kim et al, 2011) }\end{array}$ \\
\hline & & $\begin{array}{l}\text { Mi federación se ha comportado de la manera que yo esperaba } \\
\text { en las actividades en las que he participado }\end{array}$ & $\begin{array}{l}\text { (Hennig-Thurau, } \\
\text { Langer, \& Hansen, 2001) }\end{array}$ \\
\hline \multirow{2}{*}{ Felicidad } & \multirow{2}{*}{0,892} & Participar en las actividades de mi federación me hace feliz & $\begin{array}{l}\text { (Theodorakis, Kaplanidou, } \\
\text { \& Karabaxoglou, 2015) }\end{array}$ \\
\hline & & $\begin{array}{l}\text { Los federados son felices cuando participan en las actividades } \\
\text { de mi federación. }\end{array}$ & (Gong \& Yi, 2018) \\
\hline
\end{tabular}




\section{Resultados}

Los resultados se han obtenido utilizando el programa Dyane, programa estadístico creado por Santesmases (2009) para el desarrollo de encuestas en investigación comercial.

Se ha realizado un análisis ANOVA, mediante el cálculo del test F de Snedecor permite determinar si existen diferencias significativas entre los valores medios obtenidos en cada una de las categorías consideradas. A través de la prueba de ji cuadrado, realizada con 16 grados de libertad sobre la muestra, se confirma en todos los indicadores observados, la relación entre la calidad percibida la felicidad. En la tabla 2, se puede observar las conclusiones de la prueba F de Snede- cor, que nos confirman la existencia de diferencias significativas entre los distintos grupos, dado que la p valor < 0,05 al 95\% de confianza.

Seguidamente se ha realizado una prueba que analiza la varianza unidireccional de la felicidad y la calidad percibida y el resultado ha confirmado nuestro enfoca ya que se ha rechazado la hipótesis de independencia, obteniéndose como resultado de la prueba $F$ de Snedecor para 4 y 594 grados de libertad 155,2908, cuyo porcentaje de la varianza explicada fue mayor al 50\%, concretamente de un 51,12\%. En la tabla 3 se puede observar la suma de cuadrados de las variables: «Participar en las actividades de mi federación me hace feliz» $\mathrm{y}$ «Los servicios y activida-

\section{Tabla 2. Valores medios de los indicadores de la calidad percibida de acuerdo con el grado de felicidad de los deportistas federados}

\begin{tabular}{|c|c|c|c|c|c|c|c|}
\hline \multirow[t]{2}{*}{ Denominación } & \multirow{2}{*}{$\begin{array}{l}\text { Total } \\
\text { muestra }\end{array}$} & \multicolumn{5}{|c|}{ Participar en las actividades de mi federación me hace feliz. } & \multirow{2}{*}{$\begin{array}{c}\text { F de } \\
\text { Snedecor }\end{array}$} \\
\hline & & $\begin{array}{l}\text { Completo } \\
\text { desacuerdo }\end{array}$ & Desacuerdo & Indiferencia & De acuerdo & $\begin{array}{l}\text { Completo } \\
\text { de acuerdo }\end{array}$ & \\
\hline \multirow{2}{*}{$\begin{array}{l}\text { Las actividades de mi federación } \\
\text { (campeonatos, cursos, } \\
\text { entrenamientos, etc.) se desarrollan } \\
\text { de manera eficiente. }\end{array}$} & 3,8854 & 1,9556 & 2,9149 & 3,4919 & 4,1392 & 4,5044 & $\begin{array}{r}F(4,597)= \\
100,8089\end{array}$ \\
\hline & $\mathrm{n}=602$ & $n=45$ & $n=47$ & $n=124$ & $n=158$ & $n=228$ & $p=0,0000$ \\
\hline \multirow[t]{2}{*}{$\begin{array}{l}\text { Mi federación proporciona un } \\
\text { ambiente agradable para realizar } \\
\text { las actividades que organiza. }\end{array}$} & 3,9717 & 1,8444 & 2,8723 & 3,6129 & 4,2468 & 4,6256 & $\begin{array}{r}F(4,596)= \\
141,7542\end{array}$ \\
\hline & $\mathrm{n}=601$ & $n=45$ & $n=47$ & $n=124$ & $n=158$ & $n=227$ & $p=0,0000$ \\
\hline \multirow[t]{2}{*}{$\begin{array}{l}\text { Entiendo que mi federación cobra } \\
\text { un precio justo por las actividades } \\
\text { en las que yo participo. }\end{array}$} & 3,9214 & 2,2045 & 3 & 3,3852 & 4,1329 & 4,5859 & $\begin{array}{r}F(4,593)= \\
82,3918\end{array}$ \\
\hline & $\mathrm{n}=598$ & $n=44$ & $n=47$ & $n=122$ & $n=158$ & $n=227$ & $p=0,0000$ \\
\hline \multirow{2}{*}{$\begin{array}{l}\text { Los empleados de mi federación } \\
\text { me atienden de forma eficaz mis } \\
\text { consultas. }\end{array}$} & 4,0266 & 2,2889 & 3,0851 & 3,5691 & 4,2025 & 4,6886 & $\begin{array}{r}F(4,596)= \\
93,4056\end{array}$ \\
\hline & $\mathrm{n}=601$ & $\mathrm{n}=45$ & $n=47$ & $n=123$ & $n=158$ & $\mathrm{n}=228$ & $p=0,0000$ \\
\hline \multirow[t]{2}{*}{$\begin{array}{l}\text { Los servicios y actividades que } \\
\text { ofrece mi federación en global } \\
\text { los considero de calidad }\end{array}$} & 3,99 & 1,8409 & 3,0426 & 3,626 & 4,1329 & 4,7004 & $\begin{array}{r}F(4,594)= \\
164,5740\end{array}$ \\
\hline & $\mathrm{n}=599$ & $n=44$ & $n=47$ & $n=123$ & $n=158$ & $\mathrm{n}=227$ & $p=0,0000$ \\
\hline
\end{tabular}




\section{Tabla 3 - Análisis de la varianza explicada de los indicadores: «Participar en las actividades de mi federación me hace feliz" y «Los servicios y actividades que ofrece mi federación en global los considero de calidad»"}

\begin{tabular}{|l|c|c|c|c|}
\hline Grupos & No de casos & Media aritmética & Desviación estándar & Suma de cuadrados \\
\hline Total muestra & $\mathbf{5 9 9}$ & $\mathbf{3 , 7 9 6 3}$ & $\mathbf{1 , 2 3 2}$ & $\mathbf{9 0 9 , 1 5 1 9}$ \\
\hline Categorías variable tratamiento & & & \\
\hline Completo desacuerdo & 26 & 1,5 & 0,9707 & 24,5 \\
\hline Desacuerdo & 31 & 115 & 2,0968 & 1,2009 \\
\hline Indiferencia & 178 & 2,9826 & 1,1032 & 139,7097 \\
\hline De acuerdo & 249 & 3,8258 & 0,79852 \\
\hline Completo de acuerdo & & 4,6024 & 0,6989 & 113,6011 \\
\hline
\end{tabular}

Fuente: Elaboración propia

des que ofrece mi federación en global los considero de calidad».

En todas las pruebas de la ji cuadrado analizadas, la probabilidad fue 0,000 , por lo tanto, se puede afirmar que hay una relación entre las variables de la calidad percibida y la satisfacción. Además, la prueba de la F de Snedecor muestra la existencia de diferencias significativas en las diferentes tabulaciones cruzadas de los valores medios de los indicadores de satisfacción y calidad percibida.

En ambas pruebas, de la ji cuadrado con 16 grados de libertad y de la F de Snedecor se ha medido la relación entre la satisfacción y la felicidad del federado. En la primera prueba se confirma la relación existente entre las dos variables, y en la segunda prueba se perciben diferencias significativas en los valores medios de cada grupo, como podemos ver en la tabla 4 .

También hemos analizado la varianza unidireccional considerando la felicidad como variable dependiente de la satisfacción del federado, los resultados indican que la hipótesis de independencia es rechazada, por lo que se confirma el planteamiento de estudio, e incluso en este caso el porcentaje de la varianza explicada está cercano al 50\%, con un coeficiente de determinación igual a 0,4629 .

Igualmente, si observamos los resultados de las dos pruebas de las variables confianza y felicidad podemos observar que ambas están relacionadas y si, además, tenemos en cuenta los datos observados en la tabla 5, apreciaremos diferencias significativas en los diferentes grupos de la confianza y la felicidad.

Para la variable confianza, el análisis de la varianza que hemos realizado rechaza la hipótesis de independencia de la confianza sobre la felicidad como con las variables anteriores, pero además el porcentaje de la varianza explicada es del 42,85\%.

Para terminar, analizamos las variables recogidas que tienen que ver con las relaciones sociales, la primera variable analiza si los federados tienen relación personal con otros deportistas federados y en la segunda si los federados tienen amistades dentro de la federación. Las pruebas de ji cuadrado confirman visiblemente que las dos variables están vinculadas tanto con el indicador «Partici- 
Tabla 4 - Valores medios de los indicadores de satisfacción de acuerdo con el grado de felicidad de los deportistas federados

\begin{tabular}{|c|c|c|c|c|c|c|c|}
\hline \multirow[t]{2}{*}{ Denominación } & \multirow{2}{*}{$\begin{array}{l}\text { Total } \\
\text { muestra }\end{array}$} & \multicolumn{5}{|c|}{ Participar en las actividades de mi federación me hace feliz } & \multirow{2}{*}{$\begin{array}{l}\text { F de } \\
\text { Snedecor }\end{array}$} \\
\hline & & $\begin{array}{l}\text { Completo } \\
\text { desacuerdo }\end{array}$ & Desacuerdo & Indiferencia & De acuerdo & $\begin{array}{l}\text { Completo } \\
\text { de acuerdo }\end{array}$ & \\
\hline \multirow[t]{2}{*}{$\begin{array}{l}\text { Creo que es bueno } \\
\text { para mi estar } \\
\text { federado. }\end{array}$} & 4,5717 & 3,3556 & 4,1277 & 4,3468 & 4,6815 & 4,9515 & $\begin{array}{r}F(4,595)= \\
61,9795\end{array}$ \\
\hline & $n=600$ & $n=45$ & $n=47$ & $n=124$ & $\mathrm{n}=157$ & $\mathrm{n}=227$ & $p=0,0000$ \\
\hline \multirow{2}{*}{$\begin{array}{l}\text { Estoy contento de } \\
\text { estar federado en mi } \\
\text { federación. }\end{array}$} & 4,1847 & 2 & 3,383 & 3,9113 & 4,3861 & 4,793 & $\begin{array}{r}F(4,596)= \\
129,0765\end{array}$ \\
\hline & $\mathrm{n}=601$ & $n=45$ & $\mathrm{n}=47$ & $n=124$ & $n=158$ & $n=227$ & $p=0,0000$ \\
\hline \multirow{2}{*}{$\begin{array}{l}\text { Estoy satisfecho } \\
\text { con las actividades/ } \\
\text { servicios que ofrece } \\
\text { mi federación. }\end{array}$} & 3,8965 & 1,8372 & 2,9362 & 3,3871 & 4,0886 & 4,63 & $\begin{array}{r}F(4,594)= \\
130,8788\end{array}$ \\
\hline & $n=599$ & $n=43$ & $n=47$ & $n=124$ & $n=158$ & $n=227$ & $p=0,0000$ \\
\hline
\end{tabular}

Fuente: Elaboración propia

Tabla 5 - Valores medios de los indicadores de confianza de acuerdo con el grado de felicidad de los deportistas federados

\begin{tabular}{|c|c|c|c|c|c|c|c|}
\hline \multirow[t]{2}{*}{ Denominación } & \multirow{2}{*}{$\begin{array}{l}\text { Total } \\
\text { muestra }\end{array}$} & \multicolumn{5}{|c|}{ Participar en las actividades de mi federación me hace feliz. } & \multirow{2}{*}{$\begin{array}{l}\text { F de } \\
\text { Snedecor }\end{array}$} \\
\hline & & $\begin{array}{l}\text { Completo } \\
\text { desacuerdo }\end{array}$ & Desacuerdo & Indiferencia & De acuerdo & $\begin{array}{l}\text { Completo } \\
\text { de acuerdo }\end{array}$ & \\
\hline \multirow{2}{*}{$\begin{array}{l}\text { Mi federación se ha com- } \\
\text { portado de la manera } \\
\text { que yo esperaba en las } \\
\text { actividades en las que he } \\
\text { participado. }\end{array}$} & 3,9185 & 1,9556 & 2,8936 & 3,4634 & 4,1582 & 4,5965 & $\begin{array}{r}F(4,596)= \\
120,7371\end{array}$ \\
\hline & $\mathrm{n}=601$ & $\mathrm{n}=45$ & $\mathrm{n}=47$ & $n=123$ & $n=158$ & $\mathrm{n}=228$ & $p=0,0000$ \\
\hline \multirow{2}{*}{$\begin{array}{l}\text { Mi federación tiene un } \\
\text { comportamiento com- } \\
\text { prometido con los fede- } \\
\text { rados a los que prestan } \\
\text { sus actividades. }\end{array}$} & 3,9068 & 1,7111 & 2,8298 & 3,4758 & 4,1338 & 4,6404 & $\begin{array}{r}F(4,596)= \\
140,9658\end{array}$ \\
\hline & $\mathrm{n}=601$ & $\mathrm{n}=45$ & $n=47$ & $n=124$ & $\mathrm{n}=157$ & $\mathrm{n}=228$ & $p=0,0000$ \\
\hline \multirow[t]{2}{*}{$\begin{array}{l}\text { Mi federación es honesta } \\
\text { con todos federados. }\end{array}$} & 3,7663 & 1,5333 & 2,8511 & 3,377 & 3,8797 & 4,5286 & $\begin{array}{r}F(4,594)= \\
113,3821\end{array}$ \\
\hline & $\mathrm{n}=599$ & $n=45$ & $n=47$ & $n=122$ & $\mathrm{n}=158$ & $n=227$ & $p=0,0000$ \\
\hline \multirow{2}{*}{$\begin{array}{l}\text { Mi federación se preo- } \\
\text { cupa de los federados } \\
\text { a los que prestan sus } \\
\text { actividades. }\end{array}$} & 3,7629 & 1,5778 & 2,7826 & 3,2358 & 3,9557 & 4,5463 & $\begin{array}{r}F(4,594)= \\
130,2304\end{array}$ \\
\hline & $\mathrm{n}=599$ & $\mathrm{n}=45$ & $n=46$ & $n=123$ & $\mathrm{n}=158$ & $n=227$ & $p=0,0000$ \\
\hline Euente: Elaboración propia & & & & & & & \\
\hline
\end{tabular}


Tabla 6: Análisis unidireccional de la varianza explicada sobre el nivel de felicidad explicado por las relaciones sociales con otros federados

\begin{tabular}{|l|c|c|c|c|}
\hline Grupos & No de casos & Media aritmética & Desviación estándar & Suma de cuadrados \\
\hline Total muestra & $\mathbf{5 9 9}$ & $\mathbf{3 , 7 9 9 7}$ & $\mathbf{1 , 2 3 3 9}$ & $\mathbf{9 1 1 , 9 5 9 9}$ \\
\hline Categorias variable tratamiento & & & \\
\hline Completo desacuerdo & 34 & 2,4706 & 1,3982 & 66,4706 \\
\hline Desacuerdo & 44 & 2,9091 & 1,1245 & 169,6364 \\
\hline Indiferencia & 141 & 3,3404 & 1,0969 & 174,8732 \\
\hline De acuerdo & 142 & 3,7465 & 1,1097 & 217,0798 \\
\hline Completo de acuerdo & 238 & 4,458 & 0,955 & $\mathbf{6 8 3 , 7 1 9 6}$ \\
\hline
\end{tabular}

Tabla 7: Análisis unidireccional de la varianza explicada sobre el nivel de felicidad explicado por la tenencia de amistades dentro de la federación

\begin{tabular}{|l|c|c|c|c|}
\hline \multicolumn{1}{|c|}{ Grupos } & No de casos & Media aritmética & Desviación estándar & Suma de cuadrados \\
\hline \multicolumn{1}{|c|}{ Total muestra } & $\mathbf{5 9 8}$ & $\mathbf{3 , 7 9 6}$ & $\mathbf{1 , 2 3 0 3}$ & $\mathbf{9 0 5 , 1 1 0 4}$ \\
\hline Categorías variable tratamiento & & & & 29,7778 \\
\hline Completo desacuerdo & 18 & 2,1111 & 1,2862 & 22,4375 \\
\hline Desacuerdo & 16 & 3,1875 & 1,1842 & 94,0274 \\
\hline Indiferencia & 73 & 3,1644 & 1,1349 & 147,6063 \\
\hline De acuerdo & 127 & 3,3701 & 1,0781 & 448,033 \\
\hline Completo de acuerdo & 364 & 4,1813 & 1,1094 & $\mathbf{7 4 1 , 8 8 1 9}$ \\
\hline
\end{tabular}

par en las actividades de mi federación me hace feliz» como con este otro «Los federados son felices cuando participan en las actividades de mi federación».

En la tabla 6 se puede comprobar que el análisis de varianza indica la existencia de relación entre las variables sobre relaciones sociales y la felicidad, en la tabla se puede observar que las distintas medias aritméticas de cada segmento van creciendo según se pasa de acuerdo a desacuerdo. De igual forma, la prueba $F$ de Snedecor nos da un resultado $\mathrm{p}=0,0000$, el cual nos indica la existencia de diferencias significativas en las medias de los diferentes conjuntos. 
Por último, en la tabla 7 se puede observar que el análisis de varianza que contrasta el nivel de felicidad del federado con las relaciones de amistad entre los federados, el resultado obtenido es muy parecido al de la tabla anterior, de hecho, la principal diferencia reside en el coeficiente de determinación, el cual supone un 0,25 para la tabla 6 mientras que es un 0,18 para la tabla 7 .

\section{Conclusiones e implicaciones}

Los resultados obtenidos en esta investigación son determinantes, ya que todas las pruebas realizadas sobre la muestra recogida confirman la totalidad de las hipótesis propuestas en los párrafos anteriores.

Del análisis llevado a cabo sobre la variable de la calidad percibida se puede suponer que, tanto de forma directa como indirecta, la calidad percibida podría tener influencia sobre la felicidad del federado español, y que, además, la satisfacción actuaría como variable mediadora. De esta forma, se entiende que los resultados del estudio empírico coincidirían con los trabajos encontrados en el marco conceptual sobre calidad percibida y felicidad (Gong \& Yi, 2018; Sato et al., 2014; Theodorakis et al., 2015). Además, los resultados muestran que entre la satisfacción y la felicidad existe un fuerte vínculo, por lo tanto, la hipótesis sobre satisfacción es confirmada, de este análisis asumiríamos que la satisfacción tiene influencia sobre la felicidad del deportista, (Dagger \& Sweeney, 2006; Gong \& Yi, 2018; Haller \& Hadler, 2006; Sweeney et al., 2015). El servicio que proporciona la federación no solo debe ser de calidad, sino que además debe parecerlo en la oferta de actividades, en las instalaciones y especialmente en el trato de los empleados hacia los deportistas, ya que va a tener un importante peso tanto en la satisfacción como en la felicidad de estos. Los empleados son una pieza clave en la federación puesto que la forma en la que comuniquen la información es trascendental a la hora de satisfacer a los federados

Con respecto a la confianza, aunque el marco teórico no ha dado tanto frutos sobre su relación con la felicidad como lo es el de las otras dos variables, sí que existen diversos investigadores que apoyan que estas dos variables están relacionadas y que la felicidad del deportista está afectada por el nivel de confianza en la federación (Barra et al., 2018; Bartolini \& Mikucka, 2017; Tokuda et al., 2010), y además los resultados obtenidos en esta investigación coinciden con la literatura, ya que la hipótesis que se propuso sobre la influencia que genera la confianza sobre la felicidad es confirmada. En este sentido, las Juntas directivas de las federaciones podrían enfocar sus esfuerzos de comunicación en reforzar la confianza de los deportistas federados en la organización, la transparencia y la claridad en la información pueden ser buenos atributos para mejorar la confianza de los deportistas en la federación.

Por último, la hipótesis planteada sobre la relación entre las relaciones sociales y la felicidad que proporciona la actividad física y el deporte es confirmada, puesto que todos los estadísticos analizados aceptan la hipótesis de que las relaciones sociales influyen en la felicidad derivada del deporte. Para los deportistas federados en kárate españoles, el tener un trato personal con los compañeros o tener amigos dentro de la organización les aporta mayor felicidad. Esto coincide con lo revisado en el marco teórico, puesto que para muchos autores tanto las relaciones sociales de forma general (Boudon, 2002; Gilbert, 2005; Haller \& Hadler, 2006) como la tenencia de amistades (Baldassare et al., 1984; Diener \& Seligman, 2002; Gladow \& Ray, 1986) explican la felicidad de las personas. Por eso otra de las tareas de los directi- 
vos de las federaciones es la de fortalecer las relaciones entre los deportistas que participan de algún modo en la federación, promoviendo la amistad y el compañerismo, a través de campañas que refuercen la competencia sana y la deportividad, pues estas características enriquecen a las personas y son piezas claves para favorecer la felicidad de los federados (Haller \& Hadler, 2006).

Por tanto, los resultados que se reflejan en esta investigación podrán ser utilizados por los directivos de las federaciones deportivas para crear estrategias de comunicación más efectivas para conseguir aumentar el número de federados a través de la conexión entre los servicios ofrecidos por las federaciones y la maximización de la felicidad del deportista federado. Finalmente, cabe decir que, aunque esta investigación se ha llevado solo sobre una federación deportiva a nivel nacional, la federación de Karate, nuestras futuras líneas de investigación se centrarán en llevar a cabo investigaciones similares en otras federaciones deportivas a nivel nacional para corroborar los resultados conseguidos y generar nuevas perspectivas de análisis. 


\section{ANEXO 1}

\section{Cuestionario completo}

Pregunta 1. Genero:

1. Hombre

2. Mujer

\section{Pregunta 2. Edad:}

1. Menor de 16

2. De 16 a 29

3. De 30 a 44

4. De 45 a 64

5. A partir de 65

\section{Pregunta 3. Cinturón:}

1. De cinturón blanco a marrón

2. Cinturón negro $1^{\circ}$ ó $2^{\circ}$ Dan

3. Cinturón negro $3^{\circ}$ ó $4^{\circ}$ Dan

4. Cinturón negro $5^{\circ}$ ó $6^{\circ}$ Dan

$\square$ 5. Cinturón negro $7^{\circ}, 8^{\circ}, 9^{\circ}$ ó $10^{\circ}$ Dan

Pregunta 4. Años que llevo federado:

1. No estoy federado (fin de la encuesta)

2. Menos de 1 año

3. Entre 1 y 5 años

4. Entre 6 y 10 años

5. Entre 11 y 20 años

6. Más de 20 años

\section{Pregunta 5. ¿Cúal es tu federación?}

1. Madrileña

2. Andaluza

3. Aragonesa

4. Asturiana

5. Balear

6. Canaria

7. Cántabra

8. Castilla y León

9. Castilla- La Mancha

10. Catalana

11. Ceutí

\section{Extremeña}

13. Gallega

14. Melillense

15. Murciana

16. Navarra

17. Riojana

18. Valenciana

19. Vasca

Pregunta 6. Actividades en las que he participado en los últimos 12 meses en mi federación (puede responder varias opciones):

1. Competiciones y/o trofeos

$\square$ 2. Cursos

$\square$ 3. Entrenamientos

4. Examenes

$\square$ 5. Otros

$\square$ 6. Ninguna

Pregunta 7. Creo que es bueno para mi estar federado.

1. Completo desacuerdo

2. Desacuerdo

3. Indiferencia

$\square$ 4. De acuerdo

$\square$ 5. Completo de acuerdo

Pregunta 8. Estoy contento de estar federado en mi federación.

1. Completo desacuerdo

2. Desacuerdo

3. Indiferencia

4. De acuerdo

5. Completo de acuerdo

Pregunta 9. Estoy satisfecho con las actividades/servicios que ofrece mi federación.

1. Completo desacuerdo

2. Desacuerdo

3. Indiferencia 
4. De acuerdo

5. Completo de acuerdo

Pregunta 10. Las actividades de mi federación (campeonatos, cursos, entrenamientos, etc.) se desarrollan de manera eficiente.

\section{Completo desacuerdo}

2. Desacuerdo

3. Indiferencia

4. De acuerdo

5. Completo de acuerdo

Pregunta 11. Mi federación proporciona un ambiente agradable para realizar las actividades que organiza.

1. Completo desacuerdo

2. Desacuerdo

3. Indiferencia

4. De acuerdo

5. Completo de acuerdo

Pregunta 12. Entiendo que mi federación cobra un precio justo por las actividades en las que yo participo.

$\square$ 1. Completo desacuerdo

2. Desacuerdo

3. Indiferencia

4. De acuerdo

5. Completo de acuerdo

Pregunta 13. Los empleados de mi federación me atienden de forma eficaz mis consultas.

\section{Completo desacuerdo}

2. Desacuerdo

3. Indiferencia

4. De acuerdo

5. Completo de acuerdo
Pregunta 14. Los servicios y actividades que ofrece mi federación en global los considero de calidad

$\square$ 1. Completo desacuerdo

2. Desacuerdo

3. Indiferencia

4. De acuerdo

5. Completo de acuerdo

Pregunta 15. Mi federación se ha comportado de la manera que yo esperaba en las actividades en las que he participado.

$\square$ 1. Completo desacuerdo

2. Desacuerdo

3. Indiferencia

4. De acuerdo

5. Completo de acuerdo

Pregunta 16. Mi federación tiene un comportamiento comprometido con los federados a los que prestan sus actividades.

$\square$ 1. Completo desacuerdo

2. Desacuerdo

3. Indiferencia

4. De acuerdo

5. Completo de acuerdo

Pregunta 17. Mi federación es honesta con todos federados.

1. Completo desacuerdo

2. Desacuerdo

3. Indiferencia

4. De acuerdo

5. Completo de acuerdo

Pregunta 18. Mi federación se preocupa de los federados a los que prestan sus actividades.

1. Completo desacuerdo

2. Desacuerdo 
3. Indiferencia

4. De acuerdo

5. Completo de acuerdo

Pregunta 19. Participar en las actividades de mi federación me hace feliz.

1. Completo desacuerdo

2. Desacuerdo

3. Indiferencia

4. De acuerdo

5. Completo de acuerdo

Pregunta 20. Los federados son felices cuando participan en las actividades de mi federación.

$\square$ 1. Completo desacuerdo

$\square$ 2. Desacuerdo

$\square$ 3. Indiferencia

$\square$ 4. De acuerdo

$\square$ 5. Completo de acuerdo

Pregunta 21. Tengo trato personal con el resto de federados de mi federación.

1. Completo desacuerdo

2. Desacuerdo

3. Indiferencia

4. De acuerdo

5. Completo de acuerdo

Pregunta 22. Tengo amigos que pertenecen a mi federación.

1. Completo desacuerdo

2. Desacuerdo

3. Indiferencia

4. De acuerdo

5. Completo de acuerdo
Pregunta 23. Participo en la organización de cursos o eventos de mi federación.

1. Completo desacuerdo

2. Desacuerdo

3. Indiferencia

$\square$ 4. De acuerdo

5. Completo de acuerdo 


\section{Bibliografía}

Ahuvia, A. (2008). If money doesn't make us happy, why do we act as if it does? Journal of Economic Psychology, 29, 491507.

Aksoy, L., Keiningham, T. L., Buoye, A., Larivière, B., Williams, L., y Wilson, I. (2015). Does loyalty span domains? Examining the relationship between consumer loyalty, other loyalties and happiness. Journal of Business Research, 68(12), 2464-2476.

Anderson, E., Fornell, C., y Lehmann, D. (1994). Customer satisfaction, market share, and profitability: Findings from Sweden. Journal of Marketing, 58(3), 53-66.

Anderson, E., y Weitz, B. (1989). Determinants of Continuity in Conventional Industrial Channel Dyads. Marketing Science, 8(4), 310-323.

Andreasen, A. R. (1994). Social Marketing: its Definition and Domain. Journal of Public Policy y Marketing, 13(I), 108-114.

Andreassen, T. W. (2000). Antecedents to satisfaction with service recovery. European Journal of Marketing, 34(1/2), 156175 .

Argyle, M. (1994). The Psychology of Happiness. London: Routledge.

Baldassare, M., Rosenfield, S., y Rook, K. S. (1984). The types of social relations predicting elderly well-being. Research on Aging, 6(4), 549-559.

Barra, C., Pressgrove, G., y Torres, E. (2018). Trust and commitment in the formation of donor loyalty. The Service Industries Journal, 38(5-6), 360-377.

Bartolini, S., y Mikucka, M. (2017). Money, Trust and Happiness in Transition Countries: Evidence from Time Series. Social Indicators Research, 130(1), 87-106.

Berry, L. (1995). Relationship Marketing of Services Growing Interest, Emerging Perspectives. Journal of the Academy of Marketing Science, 23(4), 236-237.

Bonsón Ponte, E., Carvajal-trujillo, E., y Escobar Rodríguez, T. (2015). Influence of trust and perceived value on the intention to purchase travel online: Integrating the effects of

assurance on trust antecedents. Tourism Management, 47, 286-302.

Boudon, R. (2002). Declin de la morale? Declin des valeurs. Quebec: Editions Nota Bene.
Boulding, W., Kalra, A., Staelin, R., y Zeithaml, V. A. (1993). A dynamic process model of service quality: From expectations to behavioral intentions. Journal of Marketing Research, 30(1), $7-27$.

Campillo Álvarez, J. E. (2007). El mono obeso: la evolución humana y las enfermedades de la opulencia: diabetes, hipertensión, arteriosclerosis (2010th ed.). Barcelona: Critica.

Chaudhuri, A., y Holbrook, M. B. (2001). The chain of effects from brand trust and brand affect to brand performance: The role of brand loyalty. Journal of Marketing, 65(2), 81-93.

Consejo Superior de Deportes. (2019, 1 de mayo). Deporte de Alto Nivel y Alto Rendimiento. Disponible en: https:// www.csd.gob.es/es/alta-competicion/deporte-de-altonively-alto-rendimiento

Cuesta Valiño, P., Gutiérrez Rodríguez, P., y Núñez-Barriopedro, E. (2019). The impact of corporate social responsibility on customer loyalty in hypermarkets: A new socially responsible strategy. Corporate Social Responsability and Environmental Management, 1-9.

Dagger, T. S., y Sweeney, J. C. (2006). The Effect of Service Evaluations on Behavioral Intentions and Quality of Life. Journal of Service Research, 9(1), 3-18.

Demir, M., y Urberg, K. A. (2004). Friendship and Adjustment among Adolescents. Journal of Experimental Child Psychology, 88(1), 68-82.

Demir, M., y Weitekamp, L. A. (2007). I am so happy cause today I found my friend: friendship and personality as predictors of happiness. Journal of Happiness Studies, 8, 181211.

Deng, Z., Lu, Y., Wei, K. K., y Zhang, J. (2010). Understanding Customer Satisfaction and Loyalty: An Empirical Study of Mobile Instant Messages in China. International Journal of Information Management, 30(4), 289-300.

Diener, E., Lucas, R. E., y Oishi, S. (2002). Subjective WellBeing: The Science of Happiness and Life Satisfaction. En Handbook of positive psychology (pp. 63-73). New York: Oxford University Press.

Diener, E., y Seligman, M. E. P. (2002). Very happy people. Psychological Science, 13, 81-84.

Dwyer, F. R., Schurr, P. H., y Oh, S. (1987). Developing BuyerSeller Relationships. Journal of Marketing, 51(2), 11-27.

Easterlin, R. A. (2001). Income and Happiness: Towards a Unified Theory. The Economic Journal, 111(473), 465-484. 
Fornell, C. (1992). A National Customer Satisfaction Barometer the Swedish Experience. Journal of Marketing, 56(1), 6-21.

Garbarino, E., y Johnson, M. S. (1999). The different roles of satisfaction, trust, and commitment in customer relationships. Journal of Marketing, 63(2), 70-87.

Gefen, D., Straub, D., y Boudreau, M. (2000). Structural equation modeling and regression: Guidelines for research practice. Communications of the Association for Information Systems, 4(7), 1-78.

Gilbert, D. T. (2005). Stumbling Happiness. New York: Vintage Books.

Gladow, N. W., y Ray, M. P. (1986). The impact of informal support systems on the well-being of low income single parents. Journal of Applied Family and Child Studies, 35, 113123.

Gong, T., y Yi, Y. (2018). The effect of service quality on customer satisfaction, loyalty, and happiness in five Asian countries. Psychology y Marketing, 35, 427-442.

Gwinner, K. P., Gremler, D. D., y Bitner, M. J. (1998). Relational Benefits in Services Industries: The Customer 's Perspective. Journal of the Academy of Marketing Science, 26(2), 101-114.

Haller, M. A. X., y Hadler, M. (2006). How social relations and structures can produce happiness and unhappiness: an international comparative analysis. Social Indicators Research, 75, 169-216.

Hallmann, K., Breuer, C., y Ku, B. (2013). Happiness, pride and elite sporting success: What population segments gain most from national athletic achievements? Sport Management Review, 16, 226-235.

Hennig-Thurau, T., Gwinner, K. P., y Gremler, D. D. (2002). Understanding relationship marketing outcomes: An integration of relational benefits and relationship quality. Journal of Service Research, 4(3), 230-247.

Hennig-Thurau, T., Langer, M. F., y Hansen, U. (2001). Modeling and managing student loyalty: An approach based on the concept of relationship quality. Journal of Service Research, 3(4), 331-344.

Holbrook, M. B., y Corfinan, K. P. (1985). Quality and Value in the Consumption Experience: Phacdrus Rides Again. En Perceived Quality, Lexington. MA: Lexington Books.

Huang, H., y Humphreys, B. R. (2012). Sports participation and happiness: Evidence from US microdata. Journal of Economic Psychology, 33(4), 776-793.
Hur, Y., Ko, Y. J., y Valacich, J. (2011). A Structural Model of the Relationships Between Sport Website Quality, E-Satisfaction, and E-Loyalty. Journal of Sport Management, 25, 458-473.

Jang, W., Ko, Y. J., Wann, D. L., y Kim, D. (2017). Does Spectatorship Increase Happiness? The Energy Perspective. Journal of Sport Management, 31, 333-344.

Javalgi, R. R. G., y Moberg, C. R. (1997). Service loyalty: implications for service providers. Journal of Services Marketing, 11(3), 165-179.

Johnson, D., y Grayson, K. (2005). Cognitive and affective trust in service relationships. Journal of Business Research, 58(4), 500-507.

Khan, S., y Hussain, M. (2013). Determinants of Consumer Happiness and Its Role in Customer Loyalty. International Review of Management and Business Research, 2(1), 11-19.

Loranca Valle, C., Cuesta-Valiño, P. y Núñez-Barriopedro, E. (2019). Gestión de calidad como estrategia clave de la felicidad en el deporte federado. Retos Revista de Ciencias de la Administración y Economía, 9(18), pp. 203-218.

Lyubomirsky, S., King, L., y Diener, E. (2005). The Benefits of Frequent Positive Affect: Does Happiness Lead to Success? Psychological Bulletin, 131(6), 803-855.

Lyubomirsky, S., Sheldon, K. M., \& Schkade, D. (2005). Pursuing happiness: The architecture of sustainable change. Review of general psychology, 9(2), 111-131.

Mathur, R. (2004). Relations between friendship quality and measures of well-being. En Society for Research on Adolescence. Baltimore, MD, USA.

Maxham, J. G., y Netemeyer, R. G. (2002). A Longitudinal Study of Complaining Customers' Evaluations of Multiple Service Failures and Recovery Efforts. Journal of Marketing, 66(4), 57-71.

Moorman, C., Deshpandé, R., y Zaltman, G. (1993). Factors Affecting Trust in Market Research Relationships. Journal of Marketing, 57(1), 81-101.

Morgan, R. M., y Hunt, S. D. (1994). The Commitment- Trust Theory of Relationship Marketing. Journal of Marketing, 58(3), 20-38.

Nicolao, L., Irwin, J. R., y Goodman, J. K. (2009). Happiness for sale: Do experiential purchases make consumers happier than material purchases? Journal of Consumer Research, 36(2), 188-198. 
Oliver, R. L. (1980). A cognitive model of the antecedents and consequences of satisfaction decisions. Journal of Marketing, 17(4), 460-469.

Parasuraman, A., Zeithaml, V. A., y Berry, L. L. (1988). Servqual: A Multiple-Item Scale for Measuring Consumer Perc. Journal of Retailing, 64(1), 12.

Santesmases Mestre, M. (2009). Dyane: versión 4, diseño $y$ análisis de encuestas en investigación social y de mercados. Madrid: Pirámide

Sato, M., Jordan, J. S., y Funk, D. C. (2014). The role of physically active leisure for enhancing quality of life. Leisure Sciences, 36(3), 293-313.

Schöppe, S., Bauman, A., y Bull, F. (2004). International review of national physical activity policy. Sydney: New South Wales Center for Physical Activity and Health.

Smith, A., y Stewart, B. (2010). The special features of sport: A critical revisit. Sport Management Review, 13, 1-13.

Shin, D., y Kim, W. (2008). Forecasting customer switching intention in mobile service: An exploratory study of predictive factors in mobile number portability. Technological Forecasting and Social Change, 75(6), 854-874.

Su, L., Swanson, S. R., Y Chen, X. (2016). The effects of perceived service quality on repurchase intentions and subjective well-being of Chinese tourists: The mediating role of relationship quality. Tourism Management, 52, 82-95.

Subdirección General de Estadística y Estudios Secretaría General Técnica and Deporte (2015) Encuesta de hábitos deportivos en España 2015.
Supreme Council for Sports in Spain. Ministry of Education, Culture and Sports. (2019, 25 de febrero). Disponible en: http://www.csd.gob.es/csd/competicion

Sweeney, J. C., Danaher, T. S., y Mccoll-kennedy, J. R. (2015). Customer Effort in Value Cocreation Activities: Improving Quality of Life and Behavioral Intentions of Health Care Customers. Journal of Service Research, 18(3), 318-335.

Theodorakis, N. D., Kaplanidou, K. (KIKI), y Karabaxoglou, I. (2015). Effect of Event Service Quality and Satisfaction on Happiness Among Runners of a Recurring Sport Event. Leisure Sciences, 37, 87-107.

Tokuda, Y., Fujii, S., y Inoguchi, T. (2010). Individual and Country-Level Effects of Social Trust on Happiness: The Asia Barometer Survey. Journal of Applied Social Psychology, 40(10), 2574-2593.

Vasconcelos, A. F. (2008). Broadening even more the internal marketing concept. European Journal of Marketing, 42(11/12), 1246-1264.

Veenhoven, R. (1997). Progres dans la comprehension du bonheur. Revue Quebecoise de Psychologie, 18, 29-74.

Veenhoven, R. (2009). How do we assess how happy we are? Tenets, implications and tenability of three theories. Happiness, economics and politics, 45-69.

Wemmer, F, y Koenigstorfer, J. (2016). Open Innovation in Nonprofit Sports Clubs. International Journal of Voluntary and Nonprofit Organizations, 27(4), 1923-1949.

Zeithaml, V. A. (1987). Defining and Relating Price, Perceived Quality, and Perceived Value. MSI and Marketing Science, 87101 . 
